\title{
Laser effect on particle size of Barium Titanate nanoparticles prepared by a Sol-Gel Method
}

\author{
Massaud Mostafa $^{\text {A }}$, A. A. Ebnalwaled ${ }^{\text {B }}$, M.K.Gerges ${ }^{\mathrm{c}}$, Reham Roshdy ${ }^{\mathrm{c}}$. \\ ${ }^{a}$ Laser Tech. \& Environment Lab., Physics Department, Faculty of Science, South Valley University, Qena, \\ 83523 Egypt. \\ ${ }^{b}$ Electronics \& Nano Devices Lab., Physics Department, Faculty of Science, South Valley University, Qena, \\ 83523 Egypt. \\ c Physics Department, Faculty of Science, South Valley University, Qena, 83523 Egypt.
}

\begin{abstract}
Barium titanate, BaTiO3 nanoparticles powders were prepared by Sol-Gel Method. The hydrolysis of complex alkoxide precursor that was prepared in a reflux of metallic barium and tetraethylorthotitanate in solvent. The hydrolysis was performed by the addition of water/ethanol solution to the precursor solution. The laser diode Beam with $1000 \mathrm{~mW}$ are applied vertically on the center of refluxed row materials to obtain two types of samples one before the irradiation by laser beam and the other after. The two types of samples were annealed at $1000^{\circ} \mathrm{C}$ for different annealing time $(2,4,6,8$ hours $)$. the crystallite size was determined with $X$ ray diffractometry for all samples after and before the laser irradiation .
\end{abstract}

Keywords: Laser beam, Barium titanate, Nanocrparticles, Sol-Gel, particle size.

\section{Introduction}

Barium titanate $\left(\mathrm{BaTiO}_{3}\right)$ is one of the best known Perovskite ferroelectric compounds $(\mathrm{A} 2+\mathrm{B} 4+\mathrm{O} 3)$ that have been extensively studied [1,2],

Because of its good dielectric and ferroelectric properties, it is widely used in thermistors, multilayer ceramic capacitors (MLCs), and electro-optic devices. Recent developments in microelectronic and communication technology involve the miniaturization of MLCs. To achieve this and to make the next advance, high dielectric constant ceramic particles of better quality and small, uniform size are needed [3]

Recently nanocrystalline $\mathrm{BaTiO}_{3}$ particles have been prepared by wet chemical methods [4-7] such as sol-gel, coprecipitation, and hydrothermal methods. However, the products obtained by (co) precipitation or the sol-gel method are either amorphous or precursor compounds. Calcination at $800-1000^{\circ} \mathrm{C}$, followed by milling, is usually required to form crystalline BT powders.

According to the studies of previous investigators, the critical crystallite size varied with the preparation methods. Improvement of the dielectric properties needs to develop preparation method of the BT particles and to clarify the mechanism of crystal phase transformation[8].

The aim of this research is to prepare BT nanoparticles by the sol-gel technique and study the effect of low energy Laser beam for different annealing time on their crystallite size and to clarify its effect on their crystallite size using transmittance electron microscopy and X-ray diffractometry.

In present work, we prepared the BT nanoparticles with the sol-gel technique using alkoxide complex precursor including ethanol as a solvent, then we prepared it by same method in presence of Diode Laser applied vertically during the reflux process.

\subsection{Materials}

\section{Experimental}

Starting reagents were metallic barium (Alfa Aesar a Johnson matthey Co.) and tetraethylorthotitanate (TEOT, 99\%) (Alfa Aesar a Johnson matthey Co.). Special grade reagents (Alfa Aesar a Johnson matthey Co.) of ethanol (99.5\%) used as solvent. Water was distilled .

\subsection{Synthesis}

Barium titanate nano-particles were prepared by Sol-Gel method. The starting reagents for barium titanate precursor solution are the metal barium and titanium iso-propoxide.

A complex alkoxide of barium-titanium was prepared as a precursor of BT particles. At first, a solution $\left(10^{-4} \mathrm{~m}^{3}\right)$ that dissolved metallic barium $(10 \mathrm{mmol})$ in ethanol solvent was refluxed on magnetic starrer at $70^{\circ} \mathrm{C}$ for $1 \mathrm{~h}$. Then, TEOT $(10 \mathrm{mmol})$ was added to the solution, which was refluxed for another $2 \mathrm{~h}$, and a transparent complex alkoxide was obtained. To hydrolyze the complex alkoxide, the solution was mixed with an equal volume $\left(10^{-4} \mathrm{~m}^{3}\right)$ of an ethanol/water mixture. For the same previous procedures the material was then irradiated by diode laser with power $1000 \mathrm{~mW}$ for 1 and $2 \mathrm{hrs}$. 
The BT nano-particles were prepared by calcining the BT precursor particle at $70^{\circ} \mathrm{C}$ to remove the residual organics and kept at $70^{\circ} \mathrm{C}$ for $10 \mathrm{~h}$. The solution turned opaque, which indicated the formation of the BT particles. by the annealing at $1000^{\circ} \mathrm{C}$ for different annealing times ( $\left.2 \mathrm{~h}, 4 \mathrm{~h}, 6 \mathrm{~h}, 8 \mathrm{~h}\right)$ to deposit the BT nanoparticles with different crystallite size. The particle crystallite size was determined by XRD analysis by using Scherrer's equation.

\subsection{Measurements}

The BT particles were characterized by X-ray diffraction (XRD). The particles were observed with Zeiss LEO 912 OMEGA operated at $100 \mathrm{kV}$ of accelerating voltage. Crystal structures of the barium titanate particles were measured with an X-ray diffractometer (Rigaku RU-200A) operated at $40 \mathrm{kV}$ and 30mAwith $\mathrm{CuK} \alpha$ radiation using monochromator.

\section{Results and Discussion}

The BT particles were characterized by XRD. Fig. 1 shows the X-ray diffraction patterns of $\mathrm{BaTiO}_{3}$ samples before (A) and after (B) irradiation by laser for annealing 2 hours annealing time at $1000^{\circ} \mathrm{C}$.

From X-ray analysis the structure is change. Before laser irradiation, the diffractogram indicate the formation of cubic phase of $\mathrm{BaTiO}_{3}$. The appearance of X-ray reflections at $2 \theta=22.0,31.645,38.955,45.270$ and 56.135 is in correlation with JCPDS (31-0174) standards.

After laser irradiation, the diffractogram indicates the formation show the formation of tetragonal phase of $\mathrm{BaTiO}_{3}$, which is approved by the appearance of X-ray reflections at $2 \theta=22.184,31.49,38.849,45.152,50.729,56.075$ and 65.711 (JCPDS 05-0626). One can observe that the peaks of (110) and (111). These two peaks shifted toward lower angles for the irradiated sample by Laser. It can be considered that the phase transformation led to an increase in the lattice constants [8].

The crystallite size was calculated from Full Width at Half Maximum (FWHM) for all the peaks using Scherer formula:

$$
t=\frac{0.9 \lambda}{\beta \cos \theta}---------
$$

$\beta=$ full width at half maximum, $\theta=$ Bragg angle for the actual peak. The value of crystallite size decreases with irradiation of Laser beam only for $2 \mathrm{~h}$ annealing time .

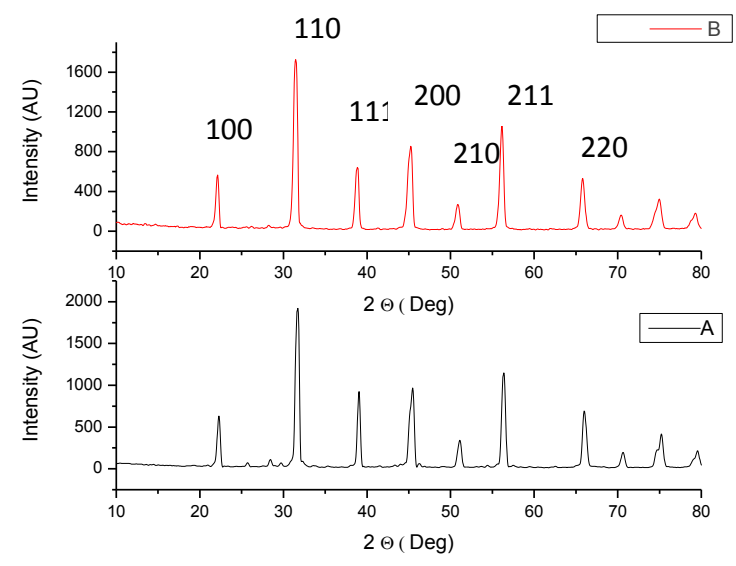

Fig.1 shows the X-ray diffraction patterns of $\mathrm{BaTiO}_{3} \mathrm{samples}$ annealed at $1000{ }^{\circ} \mathrm{C}$ for $2 \mathrm{~h}$ in air (A) before and $(B)$ after irradiation by laser.

Fig. 2 ,3,4 shows the X-ray diffraction patterns of $\mathrm{BaTiO}_{3}$ samples before (C,E,G) and after (D,F,H ) irradiation by laser for annealing $4,6,8$ hours annealing time at $1000^{\circ} \mathrm{C}$ respectively.

One can observe that there is no shift of peaks for samples before and after the irradiation by laser beam . 


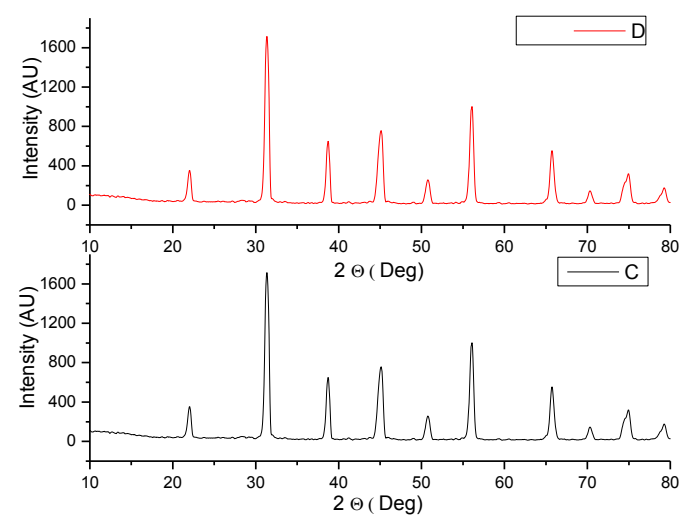

Fig.2 shows the $\mathrm{X}$-ray diffraction patterns of $\mathrm{BaTiO}_{3}$ samples annealed at $1000{ }^{\circ} \mathrm{C}$ for $4 \mathrm{~h}$ in air (C) before and (D) after irradiation by laser.

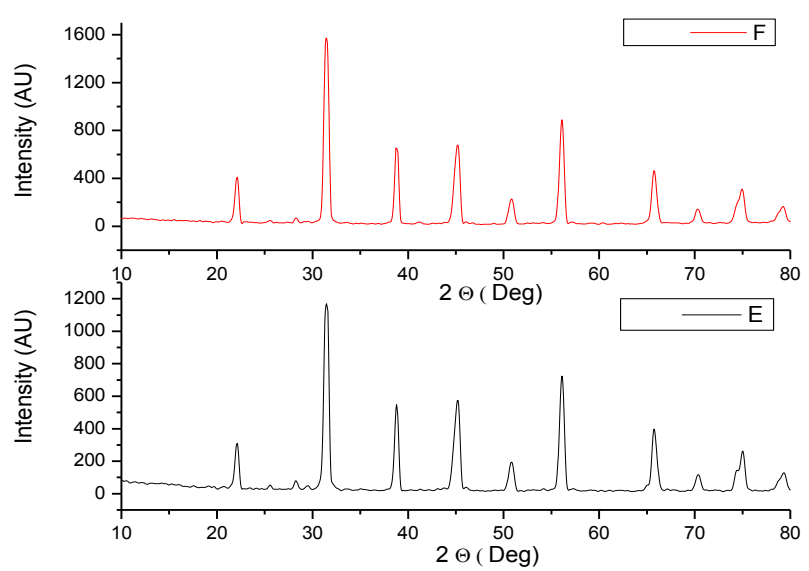

Fig.3 shows the $\mathrm{X}$-ray diffraction patterns of $\mathrm{BaTiO}_{3}$ samples annealed at $1000{ }^{\circ} \mathrm{C}$ for $6 \mathrm{~h}$ in air (E) before and (F) after irradiation by laser.

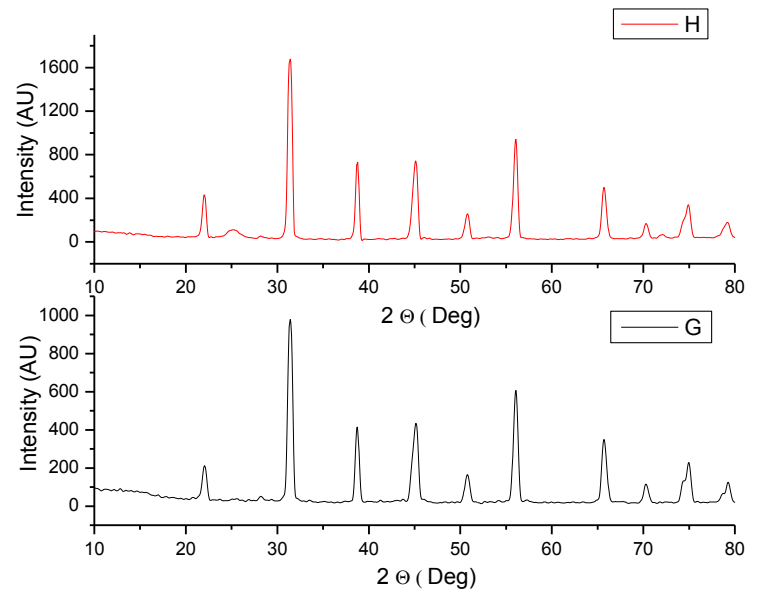

Fig.4 shows the X-ray diffraction patterns of $\mathrm{BaTiO}_{3}$ samples annealed at $1000^{\circ} \mathrm{C}$ for $8 \mathrm{~h}$ in air (G) before and (H) after irradiation by laser.

Figure 5 shows the crystallite sizes estimated from the broadening of (111) peaks for various annealing time. It is clear that for $2 \mathrm{~h}$ annealing time and after laser irradiation the crystallite size of the material decreases because of change in the lattice constant of the material, and the effect of laser irradiation on the crystallite size approximately disappeared for long annealing time. 


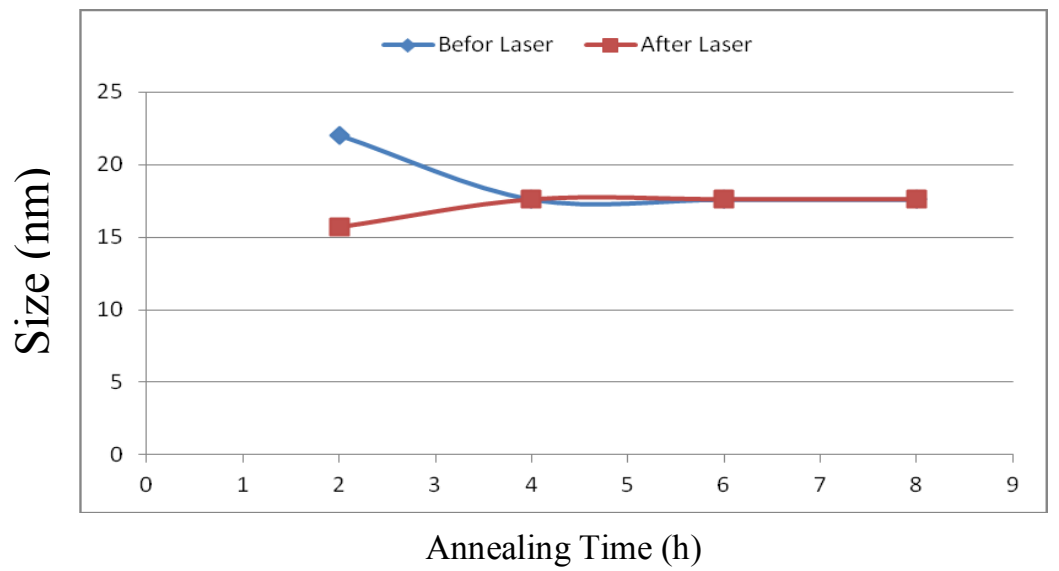

Fig.5 shows the crystallite sizes estimated from the broadening of (111) peaks for various annealing time.

\section{Conclusion}

From the above results we conclude that for $\mathrm{BaTiO}_{3}$ nanoparticles prepared by Sol-Gel method, and after laser irradiation average grain size of the material decreases as there is change in the lattice constant of the material for $2 \mathrm{~h}$ annealing time. The effect of laser irradiation on the crystallite size approximately disappeared for long annealing time.

\section{Acknowledgment}

This research was supported by South Valley University, Qena, Egypt.

\section{References:}

[1] D. Makovec, Z. Samadmija and M. Drofenik, J. Am. Ceram. Soc. 87, 1324 (2004)

[2] D. Maga, P. Igor and M. Sergei, J. Mater. Chem. 10, 941 (2000).

[3] S.Venigalla: Am. Ceram. Soc. Bull. 6 (2001) 63

[4] A. Jana, T. K. Kundu, S. K. pradhan and D. Chakravorty, J. Appl. Phys. 97 (4), 44311 (2005).

[5] Z. Jin, C. Ang and Z. Yu, J. Am. Ceram. Soc. 82(5) 1345 (1999).

[6] P. Yongping, Y. Wenhu and C. Shoutian, J. Rare Earths. 25, 154 (2005).

[7] V. J. Tennery and R. L. Cook, J. Am. Ceram. Soc. 44 (4), 186 (1961).

[8] Y. Kobayashi, A. Nishikata, T. Tavase , M. Konno, J.Sol-Gel Sc. and Tech. 29, 49-55, (2004) 